\title{
Diseño organizativo de las organizaciones no lucrativas centradas en personas con discapacidad
}

\author{
Martín-Pérez, Víctor* \\ Martín-Cruz, Natalia** \\ Estrada-Vaquero, Isabel ${ }^{* *}$
}

\section{Resumen}

El objetivo de este trabajo realizado sobre pequeñas organizaciones sin fines de lucro españolas dedicadas a la prestación de servicios a personas con discapacidad, es analizar como el diseño organizativo -en concreto, la delegación y los sistemas de recompensas extrínsecas e intrínsecas- influye sobre la transferencia de conocimiento entre sus empleados. Utilizando información de 105 organizaciones, obtenida por medio de un cuestionario postal, se contrastó el modelo estructural aplicando la técnica de mínimos cuadrados parciales. Los resultados muestran que la delegación conlleva una mayor utilización de recompensas y éstas, a su vez, favorecen la transmisión de conocimiento. Se puede concluir que, debido a la carencia de conocimiento técnico, los directivos de estas organizaciones no lucrativas deben delegar derechos de decisión en empleados cualificados y utilizar en mayor medida recompensas, tanto intrínsecas como extrínsecas, especialmente, las intrínsecas, para motivar a los empleados a que se transmitan conocimiento eficientemente.

Palabras clave: Personas con discapacidad, recompensas extrínsecas, recompensas intrínsecas, transmisión de conocimiento, organizaciones no lucrativas, diseños organizativos.

Recibido: 12-07-11. Aceptado: 18-04-12

* Doctor en Administración y Dirección de Empresas, Profesor contratado. Departamento de Organización de Empresas y Comercialización e Investigación de Mercados. Facultad de Ciencias Económicas y Empresariales. Universidad de Valladolid. España. e-mail: vmartin @eco.uva.es. Autor para la correspondencia.

** Doctora en Administración y Dirección de Empresas. Profesora Titular. Departamento de Organización de Empresas y Comercialización e Investigación de Mercados. Facultad de Ciencias Económicas y Empresariales. Universidad de Valladolid. España. e-mail: ambiela @eco.uva.es.

*** Doctoranda en Administración y Dirección de Empresas. Profesora Ayudante. Departamento de Organización de Empresas y Comercialización e Investigación de Mercados. Facultad de Ciencias Económicas y Empresariales. Universidad de Valladolid. España. e-mail: iestrada @eco.uva.es. 
Diseño organizativo de las organizaciones no lucrativas centradas en personas ...

Martín-Pérez, Víctor; Martín-Cruz, Natalia y Estrada-Vaquero, Isabel

\title{
Organizational Design of Nonprofit Organizations Focused on People with Disabilities
}

\begin{abstract}
The goal of this paper about small, Spanish nonprofit organizations providing services to people with disabilities, is to analyze how organizational design-particularly, delegation and extrinsic and intrinsic reward systems-impacts knowledge transfer among employees. Using data from 105 organizations obtained through a postal survey, the structural model was tested using the partial least squares approach. Results showed that delegation involves a greater use of rewards, and these, in turn, improve knowledge transfer. Conclusions are that, due to lack of technical knowledge, managers of these nonprofit organizations should delegate decision rights to qualified employees and utilize both extrinsic and especially intrinsic rewards to a greater degree, motivating employees to transfer knowledge efficiently.
\end{abstract}

Keywords: People with disabilities, extrinsic rewards, intrinsic rewards, knowledge transfer, nonprofit organizations, organizational designs.

\section{Introducción}

El diseño organizativo no es una tarea sencilla para ningún tipo de organización pero para las pequeñas entidades no lucrativas (ENL), a menudo carentes de recursos financieros, desarrollar un diseño eficaz y eficiente puede llegar a ser un reto si cabe más difícil y exigente. Además, los fundadores y directivos de las ENL que proporcionan servicios a las personas con alguna discapacidad -habitualmente padres $u$ otros familiares de una persona con discapacidad- suelen carecer del conocimiento técnico que requiere la atención de esa discapacidad, máxime cuando en la mayor parte de los casos la prestación requiere ser realizada por equipos integrados por profesionales con perfiles multidisciplinares (educadores sociales, médicos, psicólogos, entre otros).

Consecuencia de todo ello es que, con frecuencia, deben delegar la responsabilidad sobre las decisiones técnicas en empleados cualificados que disponen de suficiente conocimiento sobre la discapacidad. Los directivos también deben motivar a esos empleados, tanto extrínseca como intrínsecamente, para que lleven a cabo una eficaz transmisión de conocimiento que permita cumplir la misión de la ENL, consistente, en el caso que nos ocupa, en lograr la mejora de la calidad de vida de las personas con discapacidad.

A la hora de establecer el proceso delegación-motivación-transmisión de conocimiento para lograr los objetivos de la organización, los fundadores/directivos (en este tipo de ENL de reducida dimensión suele ser habitual que ambos roles estén encarnados en la misma persona) se enfrentan a dos decisiones críticas de diseño organizativo: cuánta autoridad delegar en sus empleados para lograr una mejor utilización de su conocimiento y qué sistemas de recompensas aplicar para que los empleados emprendan las acciones deseadas evitando, al mismo 
tiempo, que hagan un uso interesado de la autoridad concedida (Jensen y Meckling, 1976; Brickley, Smith y Zimmerman, 1995). La asignación de los derechos de decisión debe permitir que exista un vínculo efectivo entre la autoridad para la toma de decisiones y la acción, con la información que resulte relevante para la toma de buenas decisiones. Además, cuando se motiva a los empleados se deben tener en cuenta sus responsabilidades (Mirvis y Hackett, 1983; Leete, 2000) y su rendimiento (Balkin y Gomez-Mejia, 1990; Widener, Shackell y Demers, 2008) de modo que se puedan proporcionar recompensas adecuadas para que su comportamiento y esfuerzo se orienten a la consecución de los objetivos de la organización.

La literatura previa ha puesto de manifiesto que los sistemas de recompensas tienen un efecto positivo sobre la transmisión de conocimiento que los empleados llevan a cabo (Quigley, Tesluk, Locke y Bartol, 2007). Considerando que el recurso estratégico más importante hoy en día para las organizaciones es el conocimiento (Kogut y Zander, 1996), para las pequeñas ENL que atienden a las personas con discapacidad, en las que los empleados tienen un conocimiento especializado y complementario (ej., médicos, enfermeras, fisioterapeutas, psiquiatras, asistentes sociales, entre otros), organizar los comportamientos y esfuerzos de la organización alrededor de la transmisión de conocimiento tiene una importancia crítica para alcanzar la misión de la organización. De hecho, la transmisión de conocimiento entre los empleados de estas organizaciones es el principal método para permitirles trabajar juntos de modo efectivo y, por lo tanto, esencial para lograr la eficiencia organizativa (Huysman y de Wit, 2004; Pedraja-Rejas, Rodríguez-Ponce, y Rodríguez-Ponce, 2009). Para maximizar la transmisión de conocimiento entre los empleados, la investigación previa ha sugerido no aplicar solamente sistemas de recompensas extrínsecas (Collins y Yeager, 1988; Balkin y Gomez-Mejia, 1990; Brickley y Van Horn, 2002) sino también potenciar las recompensas intrínsecas -como la identificación con la misión y un fuerte sentido de pertenencia a la organización-que permitan a los empleados visualizar su desarrollo profesional con una mayor autonomía dentro de un buen ambiente de trabajo y en consonancia con sus valores éticos y morales (Pfeffer, 1998; Hallock, 2002).

Teniendo en cuenta el efecto de las variables organizativas (ej., delegación y recompensas extrínsecas e intrínsecas) sobre la transmisión de conocimiento, el diseño organizativo tiene un impacto significativo en la consecución de la misión $y$, por lo tanto, en la eficiencia de la organización (Brickley, Smith y Zimmerman, 1995), que es la garantía última de supervivencia de todas las organizaciones, sin que las ENL sean una excepción.

Aunque la literatura teórica ha reconocido la importancia de la delegación sobre las recompensas (Jensen y Meckling, 1992; Milgrom y Roberts, 1992) y de las recompensas, tanto intrínsecas como extrínsecas, sobre la tendencia de los empleados a transmitir conocimiento (Lucas y Ogilvie, 2006), la evidencia empírica al respecto es muy escasa.

Este trabajo realiza una contribución a la literatura sobre economía del di- 
Diseño organizativo de las organizaciones no lucrativas centradas en personas ... Martín-Pérez, Víctor; Martín-Cruz, Natalia y Estrada-Vaquero, Isabel

seño organizativo al considerar las relaciones entre delegación, recompensas y transmisión de conocimiento. En concreto, se realiza una contribución a la escasa literatura empírica que evalúa el efecto de la delegación de los derechos de decisión en empleados con conocimiento especializado sobre el diseño de los sistemas de recompensas y la influencia de estos sobre la transmisión de conocimiento entre los empleados, trasladando los argumentos de estudios empíricos previos (O'Connor, Deng y Luo, 2006; Widener, Shackell y Demers, 2008) a un ámbito nuevo, como son las ENL dedicadas a prestar servicios a personas con discapacidad. Aunque se es consciente de la existencia de estudios previos que han analizado la discapacidad en el ámbito laboral (Stone y Colella 1996; Colella 2001), el interés es sustancialmente diferente, ya que el estudio se centro en el diseño organizativo de ENL que emplean personal especializado que trabaja para mejorar la calidad de vida de las personas con discapacidad.

Para alcanzar este objetivo, se llevó a cabo un estudio en dos etapas utilizando información de las 312 ENL dedicadas a la discapacidad en Castilla y León (la región española más importante en términos de extensión), registradas en la base de datos de la Gerencia de Servicios Sociales de la Junta de Castilla y León (gobierno regional). En la primera fase, se envió un cuestionario postal dirigido al director general, utilizando la información de contacto obtenida a partir de la base de datos proporcionada por la Junta de Castilla y León. El cuestionario solicitaba el nombre de la organización, número y tipo de empleados, y discapacidad/es atendida/s. En la segunda fase, se envió un nuevo cuestionario a aquellos directivos que habían respondido al cuestionario inicial, solicitando información relacionada con variables organizativas (delegación, recompensas y transmisión de conocimiento). Ambas fases de recogida de información, desarrolladas entre abril y julio de 2007, proporcionaron un total de 105 cuestionarios válidos, lo que representa una tasa de respuesta del $34 \%$.

Con base en estudios empíricos previos, se midieron las variables -delegación, recompensas extrínsecas, recompensas intrínsecas y transmisión de conocimiento-por medio de varios ítems, valorados en escalas Likert de 5 niveles, adaptados a las ENL.

Para contrastar las hipótesis formuladas en el estudio se aplicó la técnica de mínimos cuadrados parciales (PLS). Con esta técnica, los parámetros estructurales y de medida son estimados a través de un procedimiento iterativo que combina regresiones simples y múltiples por medio de mínimos cuadrados ordinarios (OLS), de modo que evita realizar supuestos sobre la distribución de las variables observadas. Además, debido a la naturaleza parcial de esta metodología, donde los parámetros del modelo son estimados en bloques, el tamaño muestral requerido por PLS es mucho menor. El modelo planteado incluye tanto variables latentes, medidas con indicadores reflectivos, como emergentes -formativos-. El modelo fue estimado utilizando SmartPLS 2.00 M3 (Ringle, Wende y Hill, 2005). Dado que los test paramétricos tradicionales son inapropiados cuando no se han realizado supuestos sobre la distribución de las variables observadas, 
el nivel de significación estadística de los coeficientes, tanto del modelo estructural como del modelo de medida, fue determinado a través de un proceso bootstrap de re-muestreo (se generaron de forma aleatoria 500 submuestras). La evaluación estructural se llevó a cabo examinando el tamaño y la significación de los coeficientes y los valores del $\mathrm{R}^{2}$ de las variables dependientes. Los resultados obtenidos muestran que la delegación conlleva una mayor utilización de recompensas y éstas, a su vez, favorecen la transmisión de conocimiento, aspecto que favorece la consecución del objetivo de la misión de la organización que no es otra que mejorar los servicios que se prestan a las personas con discapacidad.

\section{Delegación y sistemas de recompensas en las ENL centradas en personas con discapacidad}

La literatura previa ha puesto de manifiesto que el rendimiento de una organización depende de ubicar la autoridad para tomar decisiones con el conocimiento requerido para adoptar esas decisiones (Hayek, 1945; Jensen y Meckling, 1992; Christie, Joye y Watts, 2003; Martín-Pérez, Martín-Cruz y Hernangómez Barahona, 2007; 2010). Los derechos de decisión y el conocimiento se pueden relacionar bien transfiriendo los derechos de decisión a la persona con el conocimiento relevante o bien transmitiendo el conocimiento requerido a la persona que tiene los derechos de decisión. Ambas alternativas conllevan costes. La primera genera costes de control, mientras que la segunda da lugar a los costes de transmi- sión de conocimiento que incluyen no sólo la transferencia del conocimiento a la persona que tiene los derechos de decisión, sino también los retrasos producidos en el proceso de transmisión y las pérdidas derivadas de la falta de comprensión del conocimiento que el decisor recibe para aplicarlo de forma oportuna (Jensen y Meckling, 1992; Christie, Joye y Watts, 2003). Cuando los costes de transmisión son elevados -ya sea por la especificidad o por la naturaleza tácita del conocimiento- asignar derechos de decisión a niveles inferiores de la jerarquía organizativa puede ayudar a contener los costes. Al delegar autoridad, se puede hacer un uso más eficaz del conocimiento requerido al tiempo que se evitan los costes asociados con la obtención y transmisión de ese conocimiento a los niveles jerárquicos superiores (Jensen y Meckling, 1992; Christie, Joye y Watts, 2003; Martín-Pérez, Martín-Cruz y Hernangomez Barahona 2007; 2010).

Aunque la delegación de derechos de decisión puede reducir los costes de obtención y transmisión de la información, crea un problema de riesgo moral, ya que la delegación no garantiza que los empleados actúen persiguiendo los intereses de la organización, lo que hace necesario dotarse de un marco apropiado de control. Las recompensas, basadas en el rendimiento de los empleados, son una forma de mitigar el problema de riesgo moral y alinear los intereses individuales con los de la organización (Jensen y Meckling, 1992; Christie, Joye y Watts, 2003; O'Connor, Deng y Luo, 2006; Widener, Shackell y Demers, 2008).

En las ENL, los directivos, al igual que en una empresa, transferirán los de- 
Diseño organizativo de las organizaciones no lucrativas centradas en personas ... Martín-Pérez, Víctor; Martín-Cruz, Natalia y Estrada-Vaquero, Isabel

rechos de decisión allí donde esté el conocimiento, siempre y cuando los costes de transferencia -derivados del diseño del sistema de recompensas- no excedan los beneficios de la concesión de tales derechos (Hayek, 1945; Jensen y Meckling, 1992; Christie, Joye y Watts, 2003; Martín-Pérez, Martín-Cruz y Hernangomez-Barahona 2007; 2010). De este modo, se plantea como primera hipótesis:

$\boldsymbol{H}_{1}$ : Un mayor nivel de delegación se relaciona positivamente con el uso de sistemas de recompensas.

En las ENL, esta primera hipótesis se debe desdoblar en los dos tipos de recompensas ya que los esquemas de recompensas se alejan del supuesto oportunista y de desconfianza hacia la persona que prima en la empresa, puesto que se espera que estas organizaciones atraigan directivos menos preocupados por los beneficios (Young y Finch, 1977; Hansmann, 1980) y que la mayoría de los individuos que las eligen para trabajar valoren, en mayor medida, el altruismo y las recompensas no monetarias que las recompensas financieras (Rose-Ackerman, 1986; Preston, 1989), estando dispuestos a donar parte de su esfuerzo (Hansmann, 1980; Preston, 1989). Como resultado, los sistemas de recompensas en el tercer sector son muy amplios y dan una mayor importancia a las recompensas intrínsecas (Mirvis y Hackett, 1983; Theuvsen, 2004), por lo que, en las ENL, al lado de las recompensas extrínsecas -las tradicionalmente consideradas por la literatura- se deben tener muy presentes las intrínsecas.

Como se acaba de exponer, las recompensas de los empleados de las ENL tienden a tener un mayor componente intrínseco siendo algunas de las razones argumentadas que los niveles salariales están por debajo de la media del mercado (Mirvis y Hackett, 1983; Preston, 1989; Handy y Katz, 1998; Ruhm y Borkoski, 2003) y para los empleados del tercer sector las consecuencias sociales de su trabajo son más importantes que el dinero que ganan (Mirvis y Hackett 1983; Handy y Katz, 1998; Leete, 2000). Estudios previos han señalado que los trabajadores de las ENL aceptan menores salarios a cambio de una serie de beneficios, tales como, perspectiva de un empleo estable, horario flexible (Delaney y Mark, 1996; De Gieter, De Cooman, Pepermans, Caers, Du Bois y Jegers, 2006; Tortia, 2008) y menor ritmo de trabajo (Hallock, 2002). Las recompensas intrínsecas han sido consideradas como la base para desarrollar una confianza mutua que refuerce la cohesión y la identificación de los empleados con la organización (Young, 1983; Leete, 2000), de modo que éstos estén dispuestos a utilizar su conocimiento para lograr los objetivos de la organización. Por todo ello, se propone la siguiente subhipótesis en relación con las recompensas intrínsecas:

$\boldsymbol{H}_{1 a}$ : Un mayor nivel de delegación se relaciona positivamente con la utilización de recompensas intrínsecas.

Tal y como se ha argumentado, a pesar de que las ENL hagan uso de las recompensas intrínsecas, la creciente importancia del tercer sector en las economías modernas y en el mercado laboral, con un peso cada vez mayor en el empleo del sector servicios, aumenta la probabilidad de atraer individuos menos comprometidos, lo que puede dar lugar a 
la aparición de problemas de riesgo moral $^{1}$ (Handy y Katz, 1998; Preyra y Pink, 2001). Para reducir estos problemas y alinear los intereses de esos empleados menos motivados intrínsecamente con los objetivos de la organización y teniendo en cuenta que las entidades no lucrativas tienen, por término medio, una menor estructuración jerárquica (Barnabé y Burns, 1994) y recurren en menor medida al control directo que las empresas, es necesario recurrir a las recompensas extrínsecas. Esto permite incentivar a los trabajadores a utilizar sus habilidades y conocimientos en beneficio de la organización. De este modo, se propone la siguiente subhipótesis en relación con las recompensas extrínsecas:

$\boldsymbol{H}_{1 b}$ : Un mayor nivel de delegación se relaciona positivamente con la utilización de recompensas extrínsecas.

\section{Sistemas de recompensas y transmisión de conocimiento en las ENL centradas en personas con discapacidad}

Quigley, Tesluk, Locke y Bartol (2007) acentúan la necesidad de considerar tanto las recompensas intrínsecas como las extrínsecas cuando se aborda la cuestión de la transmisión de conocimiento. Aunque las recompensas extrínsecas desempeñan un papel fundamental en la motivación de los empleados, la transmisión de conocimiento es un proceso social de intercambio que, en las ENL, habitualmente, se amplía para incluir creencias e ideales intrínsecamente mantenidos (Bock, Zmud, Kim y Lee, 2005). Como previamente se ha argumentado, la mayoría de los empleados de las ENL desean algo más de sus trabajos que la mera compensación extrínseca, por lo que los directivos de estas organizaciones pueden recurrir a las recompensas intrínsecas para apoyar los procesos de autoselección y de atracción de empleados comprometidos (Callen y Falk, 1993; Handy y Katz, 1998; Roomkin y Weisbrod, 1999). Además, los empleados de las ENL pueden estar intrínsicamente motivados para compartir su conocimiento como consecuencia de sus propios procesos de aprendizaje individual (Huysman y de Wit, 2004) o bien porque esperan o anticipan reciprocidad, esto es, que otros estén dispuestos a compartir conocimientos que les puedan ser de utilidad (Hendriks, 1999). Tampoe (1993) señala que los individuos con elevados niveles de educación y habilidades especializadas, a la hora de combinarlas para solucionar problemas y responder a nuevos desafíos, están más motivados por factores, tales como, el crecimiento personal, la autonomía operativa o el éxito en la tarea; y no tanto por recompensas financieras. Todo ello, pone de manifiesto la considerable influencia que la motivación intrínseca ejerce sobre la transmi-

1 En el tercer sector la selección adversa -el intento por filtrar al personal no comprometido- ha tenido una mayor consideración que el riesgo moral -tratar de evitar que los empleados no desarrollen comportamientos oportunistas- (Handy y Katz 1998; Steinberg 1990). 
Diseño organizativo de las organizaciones no lucrativas centradas en personas ... Martín-Pérez, Víctor; Martín-Cruz, Natalia y Estrada-Vaquero, Isabel

sión de conocimiento, al mejorar la predisposición del individuo a poner en común su conocimiento con otros miembros de la organización, facilitando así los procesos de aprendizaje.

Las recompensas intrínsecas son, por tanto, una poderosa herramienta para superar algunas de las barreras que dificultan la transmisión de conocimiento. En concreto, las recompensas intrínsecas favorecen el desarrollo de grupos informales al margen de las estructuras formales, lo cual permite, entre otras cosas, la rápida resolución de problemas, la transferencia de las mejores prácticas y el desarrollo de habilidades profesionales (Kofman y Senge, 1993). Además, las recompensas intrínsecas permiten crear un adecuado ambiente laboral que facilita la comunicación, tanto formal como informal, lo que redunda en una mayor transmisión y adquisición de nuevo conocimiento y en el desarrollo de comportamientos que apalancan el aprendizaje organizativo (Slater y Narver, 1995). Un beneficio más de las recompensas intrínsecas es que incrementan el compromiso organizativo de los trabajadores, al ayudar a que éstos tengan interés en mejorar para poder apoyar a su organización, favoreciéndose el desarrollo de capacidades para "aprender a aprender" (Swieringa y Wierdsma, 1992).

En función de lo expuesto, se puede afirmar que las recompensas intrínsecas desempeñan dos importantes funciones en el proceso de transmisión de conocimiento. Por una parte, son un resultado del propio proceso y, por otra, contribuyen y fomentan la participación de los empleados en el proceso de transmisión de conocimiento (Lucas y Ogilvie, 2006).
Por todo lo anterior, se plantea la siguiente hipótesis:

$\boldsymbol{H}_{2 \mathbf{a}}$ : Un mayor empleo de recompensas intrínsecas se relaciona positivamente con una mayor transmisión de conocimiento entre los empleados.

Los argumentos expuestos no están reñidos con los que reconocen que las recompensas extrínsecas también favorecen que los empleados realicen tareas valiosas para la organización (Milgrom y Roberts, 1992; Prendergast, 1999; Bonner y Sprinkle, 2002); no obstante, la literatura no es unánime sobre la eficacia de este tipo de recompensas sobre la transmisión de conocimiento. Si bien Lucas y Ogilvie (2006) destacan que la investigación previa sobre transmisión de conocimiento y recompensas extrínsecas sugiere una relación positiva y significativa entre estas variables, los resultados de su estudio no respaldan esta relación. Bock, Zmud, Kim, y Lee, (2005) encuentran que las recompensas extrínsecas ejercen un efecto negativo sobre la predisposición del individuo a compartir conocimiento y que las recompensas organizativas esperadas no influyen significativamente sobre las intenciones y actitudes comportamentales relacionadas con la transmisión de conocimiento. Por su parte, Osterloh y Frey (2000) señalan que la generación y transmisión de conocimiento son más importantes para empleados intrínsecamente motivados que para aquellos extrínsecamente motivados. Finalmente, Burgess (2005) muestra que los empleados que perciben mayores recompensas extrínsecas por compartir conocimiento dedican más horas a dicha tarea, incluso fuera de su equipo de trabajo. 
De hecho, algunas organizaciones han diseñado sistemas de recompensas dirigidos, específicamente, a fomentar que los empleados compartan su conocimiento entre sí (Bartol y Srivastava, 2002) porque la disposición para compartir suele depender de la reciprocidad. Por consiguiente, los empleados que se sienten suficientemente recompensados desarrollarán un compromiso más fuerte con la organización, permanecerán en ella periodos de tiempo más prolongados y crearán y transmitirán conocimiento entre ellos, lo que redunda en un mejora de su rendimiento. Las recompensas extrínsecas también desempeñan un papel en los mecanismos que favorecen la transmisión de conocimiento en el sentido de que la justicia del sistema compensatorio ayuda a desarrollar confianza entre el individuo y la organización (Bartol y Srivastava, 2002). Por tanto, se espera una relación positiva entre las recompensas extrínsecas y la transmisión de conocimiento, puesto que los empleados necesitan sentir que la organización les proporciona algo concreto y valioso -algo que les brinde calidad de vida y que complemente el sentimiento de pertenencia y reconoci- miento-. Como resultado, se propone la siguiente hipótesis:

$\boldsymbol{H}_{2 b}$ : Un mayor empleo de recompensas extrínsecas se relaciona positivamente con una mayor transmisión de conocimiento entre los empleados.

\section{Diseño organizativo y su influencia en la transmisión de conocimiento}

La información recabada por medio de los cuestionarios válidos respondidos por los 105 directores generales de pequeñas ENL dedicadas a la prestación de servicios a personas con discapacidad que componen la muestra permite contrastar las hipótesis planteadas. Las variables empleadas en el análisis -delegación, recompensas extrínsecas, recompensas intrínsecas y transmisión de conocimiento- se hicieron operativas utilizando medidas basadas en estudios empíricos previos y, siempre que fue posible, cuantificadas por medio de varios ítems, valorados en escalas Likert de 5 niveles, adaptados a las ENL, como puede verse en el Cuadro 1.

Cuadro 1

Forma de medida de las variables

\begin{tabular}{|c|c|}
\hline Autor & Delegación \\
\hline \multirow[t]{4}{*}{$\begin{array}{l}\text { Gordon y Narayanan (1984), O'Connor, Deng y } \\
\text { Luo (2006) }\end{array}$} & $\begin{array}{l}\text { Identificar actividades potenciales para la asis- } \\
\text { tencia y/o inserción social de los usuarios (DEL1) }\end{array}$ \\
\hline & $\begin{array}{l}\text { Implementar nuevos proyectos de asistencia y/o } \\
\text { inserción social (DEL2) }\end{array}$ \\
\hline & $\begin{array}{l}\text { Asignación de recursos a proyectos y realiza- } \\
\text { ción de diferentes actividades (DEL3) }\end{array}$ \\
\hline & $\begin{array}{l}\text { Evaluar el impacto de las actividades desarrolla- } \\
\text { das y realizar propuestas de mejora (DEL4) }\end{array}$ \\
\hline
\end{tabular}


Diseño organizativo de las organizaciones no lucrativas centradas en personas ...

Martín-Pérez, Víctor; Martín-Cruz, Natalia y Estrada-Vaquero, Isabel

\section{Cuadro 1 (Continuación)}

\begin{tabular}{|c|c|}
\hline Autor & Recompensas extrínsecas \\
\hline $\begin{array}{l}\text { Delaney y Mark (1996), De Gieter et al. (2006), } \\
\text { Tortia (2008), Martín-Cruz, Martín-Pérez y Tre- } \\
\text { villa-Cantero (2009), Martín-Pérez, Martín-Cruz } \\
\text { y Hernangomez-Barahona (2007, 2010) }\end{array}$ & Estabilidad y continuidad en el trabajo (EREC1) \\
\hline $\begin{array}{l}\text { De Gieter et al. (2006), Martín-Cruz y Hernango- } \\
\text { mez-Barahona }(2007,2010)\end{array}$ & $\begin{array}{l}\text { Oportunidades de formación ofrecidas por la or- } \\
\text { ganización (EREC2) }\end{array}$ \\
\hline $\begin{array}{l}\text { Collins y Yeager (1988), Balkin y Gomez-Mejia } \\
\text { (1990), De Gieter et al. (2006), Martín-Pérez, } \\
\text { Martín-Cruz y Hernangomez-Barahona (2007, } \\
\text { 2010) }\end{array}$ & $\begin{array}{l}\text { Financiación de viajes, dietas y otros gastos re- } \\
\text { lacionados con la actividad (EREC3) }\end{array}$ \\
\hline $\begin{array}{l}\text { Collins y Yeager (1988), Hallock (2002), De Gie- } \\
\text { ter et al. (2006), Tortia (2008) }\end{array}$ & $\begin{array}{l}\text { Flexibilidad para establecer el horario de trabajo } \\
\text { (EREC4) }\end{array}$ \\
\hline $\begin{array}{l}\text { Balkin y Gomez-Mejia (1990), Brickley y Van } \\
\text { Horn (2002), De Gieter et al. (2006), Martín-Pé- } \\
\text { rez, Martín-Cruz y Hernangomez - Barahona } \\
(2007,2010)\end{array}$ & Incrementos del salario fijo (EREC5) \\
\hline Autor & Recompensas intrínsecas \\
\hline
\end{tabular}

De Gieter et al., (2006), Tortia (2008), Martín- La actividad en la organización le permite crecer Cruz y Trevilla Cantero (2009) como persona, mejorar su autoconfianza y autoestima (IREC1)

Handy y Katz (1998), Leete (2000), Hallock Su actividad en la organización se ajusta a sus (2002) valores éticos y morales (IREC2)

Pfeffer (1998), De Gieter et al. (2006), Tortia El ambiente organizativo en el que desarrolla su (2008) actividad es cooperativo, fomenta el respeto mutuo y la confianza (IREC3)

Tyler (2003), Tortia (2008), Martín-Cruz y Trevil- La organización es honesta y justa en lo referenla Cantero (2009) te a métodos/procedimientos internos de gestión (IREC4)

Leete (2000), De Gieter et al. (2006), Martín- Tiene sentimiento de pertenencia a la organizaCruz y Trevilla Cantero (2009) ción -se considera parte de la organización, leal e implicado en la misma, entre otros (IREC5) 


\section{Cuadro 1 (Continuación)}

\begin{tabular}{|c|c|}
\hline Autor & Transmisión de conocimiento \\
\hline \multirow{7}{*}{$\begin{array}{l}\text { Zárraga y Bonache (2003), Bock et al. (2005), } \\
\text { Ko, Kirsch y King (2005) }\end{array}$} & $\begin{array}{l}\text { Los empleados comparten activamente ideas y } \\
\text { opiniones (KTRS1) }\end{array}$ \\
\hline & $\begin{array}{l}\text { Se toman en consideración las opiniones de } \\
\text { otros empleados antes de tomar decisiones im- } \\
\text { portantes (KTRS2) }\end{array}$ \\
\hline & $\begin{array}{l}\text { Se comparten activamente conocimientos y ex- } \\
\text { periencias entre los empleados (KTRS3) }\end{array}$ \\
\hline & $\begin{array}{l}\text { Se mantienen relaciones entre los empleados } \\
\text { que favorecen una mejor comprensión de objeti- } \\
\text { vos, métodos de trabajo, entre otros (KTRS4) }\end{array}$ \\
\hline & $\begin{array}{l}\text { Los empleados mantienen contacto frecuente } \\
\text { (e-mail, teléfono.) (KTRS5) }\end{array}$ \\
\hline & $\begin{array}{l}\text { Se utilizan manuales, informes y la información } \\
\text { requerida para mejorar el rendimiento en el tra- } \\
\text { bajo (KTRS6) }\end{array}$ \\
\hline & $\begin{array}{l}\text { Los empleados comparten documentos e infor- } \\
\text { mación (KTRS7) }\end{array}$ \\
\hline
\end{tabular}

Fuente: Elaboración propia.

Todos los ítems referentes a recompensas intrínsecas tienen una media por encima de 4 (véase Tabla 1), sustancialmente mayor que la media obtenida para las recompensas extrínsecas, lo que viene a reforzar los resultados obtenidos por estudios previos que ponen de manifiesto que, para el personal de estas organizaciones, las recompensas intrínsecas tienen mayor importancia y poder motivador que las extrínsecas.

Por lo que respecta a los ítems del constructo 'transmisión de conocimiento', también medidos en una escala Likert de 1 a 5 , todos ellos muestran valores (véase Tabla 1) considerablemente por encima de 4, atestiguando que estas organizaciones, en las que el conocimiento es básico para poder dar una respuesta apropiada a las complejas necesidades que presentan las personas con discapacidad a las que se dirigen, han realizado un importante esfuerzo para que el conocimiento de sus profesionales sea puesto en común y, de este modo, puedan prestar servicios de calidad a sus usuarios.

Finalmente, los ítems de la variable delegación, aunque tienen unos valores medios inferiores a las variables antes expuestas, todos ellos están por encima del valor 3 , prueba de que estas organizaciones están implementando sistemas de gestión más participativos en los que se 
Diseño organizativo de las organizaciones no lucrativas centradas en personas ...

Martín-Pérez, Víctor; Martín-Cruz, Natalia y Estrada-Vaquero, Isabel

Tabla 1

Estadísticos descriptivos y validez convergente

\begin{tabular}{rrrrrr}
\hline & Media & S.D. & Cargas & Pesos & Estadístico t \\
\hline Delegación & & & & & \\
DEL1 & 3,88 & 0,88 & - & 0,97 & 0,0003 \\
DEL2 & 3,78 & 0,97 & - & 0,43 & 0,0001 \\
DEL3 & 3,59 & 1,05 & - & $-0,13$ & 0,0000 \\
DEL4 & 3,85 & 0,89 & - & $-0,28$ & 0,0001
\end{tabular}

Recompensas Extrínsecas

$\begin{array}{llllll}\text { EREC1 } & 3,37 & 1,27 & - & 0,12 & 0,0004 \\ \text { EREC2 } & 2,65 & 1,33 & - & -0,075 & 0,0001 \\ \text { EREC3 } & 3,42 & 1,25 & - & 0,027 & 0,0000 \\ \text { EREC4 } & 3,93 & 1,21 & - & 0,63 & 0,0018 \\ \text { EREC5 } & 3,89 & 1,29 & - & 0,32 & 0,0008\end{array}$

Recompensas Intrínsecas

$\begin{array}{llllll}\text { IREC1 } & 4,32 & 0,77 & - & -0,39 & 0,0002 \\ \text { IREC2 } & 4,43 & 0,71 & - & 0,35 & 0,0001 \\ \text { IREC3 } & 4,14 & 0,87 & - & 0,17 & 0,0001 \\ \text { IREC4 } & 4,01 & 0,98 & - & 0,72 & 0,0016 \\ \text { IREC5 } & 4,56 & 0,67 & - & 0,30 & 0,0004\end{array}$

Transmisión de conocimiento $(\rho \mathrm{c}=0.98, \mathrm{AVE}=0.91)$

\begin{tabular}{|c|c|c|c|c|c|}
\hline KTRS1 & 4,47 & 0,65 & 0,98 & - & 106,5900 \\
\hline KTRS2 & 4,48 & 0,63 & 0,98 & - & 106,5890 \\
\hline KTRS3 & 4,53 & 0,65 & 0,96 & - & 31,6027 \\
\hline KTRS4 & 4,38 & 0,80 & 0,98 & - & 106,5902 \\
\hline KTRS5 & 4,16 & 0,92 & 0,95 & - & 28,7672 \\
\hline KTRS6 & 4,20 & 0,87 & 0,93 & - & 25,1972 \\
\hline KTRS7 & 4,21 & 0,85 & 0,90 & - & 19,0160 \\
\hline
\end{tabular}


intenta aprovechar el potencial que atesora el personal cualificado que en ellas desarrolla su actividad.

Siguiendo el enfoque de dos etapas propuesto por Anderson y Gerbing (1988), antes de contrastar y valorar el modelo estructural, se analizó tanto la fiabilidad de los ítems reflectivos para la variable transmisión de conocimiento y el constructo correspondiente, como la validez convergente y discriminante de nuestras medidas (Rodríguez-Pinto Rodríguez-Escudero y Gutiérrez-Cillán, 2008). Como se puede observar en la Tabla 2, las cargas de los ítems reflectivos para la transmisión de conocimiento son significativas y mayores de 0.6. La fiabilidad compuesta fue evaluada utilizando la me-

Tabla 2 Validez discriminante (Cross-Loadings)

\begin{tabular}{|c|c|c|c|c|}
\hline & DEL & EREC & IREC & KTRS \\
\hline DEL1 & 0.99 & 0.56 & 0.46 & 0.69 \\
\hline DEL2 & 0.96 & 0.55 & 0.44 & 0.67 \\
\hline DEL3 & 0.92 & 0.53 & 0.42 & 0.65 \\
\hline DEL4 & 0.92 & 0.52 & 0.44 & 0.65 \\
\hline EREC1 & 0.52 & 0.89 & 0.40 & 0.52 \\
\hline EREC2 & 0.38 & 0.79 & 0.40 & 0.53 \\
\hline EREC3 & 0.42 & 0.82 & 0.40 & 0.53 \\
\hline EREC4 & 0.56 & 0.98 & 0.44 & 0.59 \\
\hline EREC5 & 0.52 & 0.96 & 0.46 & 0.60 \\
\hline IREC1 & 0.27 & 0.26 & 0.60 & 0.43 \\
\hline IREC2 & 0.33 & 0.33 & 0.73 & 0.52 \\
\hline IREC3 & 0.34 & 0.34 & 0.73 & 0.52 \\
\hline IREC4 & 0.44 & 0.43 & 0.95 & 0.68 \\
\hline IREC5 & 0.27 & 0.27 & 0.61 & 0.43 \\
\hline KTRS1 & 0.65 & 0.56 & 0.73 & 0.98 \\
\hline KTRS2 & 0.65 & 0.56 & 0.73 & 0.98 \\
\hline KTRS3 & 0.63 & 0.56 & 0.69 & 0.96 \\
\hline KTRS4 & 0.65 & 0.56 & 0.73 & 0.98 \\
\hline KTRS5 & 0.64 & 0.56 & 0.66 & 0.95 \\
\hline KTRS6 & 0.70 & 0.64 & 0.63 & 0.93 \\
\hline KTRS7 & 0.71 & 0.61 & 0.60 & 0.90 \\
\hline
\end{tabular}

Nota: $\mathrm{DEL}=$ delegación. $\mathrm{EREC}=$ recompensas extrínsecas. $\mathrm{IREC}=$ recompensas intrínsecas. $\mathrm{KTRS}=$ transmisión de conocimiento.

Fuente: Elaboración propia. 
Diseño organizativo de las organizaciones no lucrativas centradas en personas ...

Martín-Pérez, Víctor; Martín-Cruz, Natalia y Estrada-Vaquero, Isabel

dida de consistencia interna $\left(\rho_{c}\right)$ desarrollada por Fornell y Larcker (1981) y la varianza media extraída (AVE) de cada constructo latente. El constructo reflectivo verifica las condiciones de $\rho_{c}$ mayor de 0.7 y AVE superior a 0.5 .

La validez discriminante se obtuvo calculando las cross-loadings. Se verifica que cada uno de los ítems reflectivos carga más en el constructo que intenta medir que en cualquier otro constructo, y cada variable latente se relaciona más con sus propias variables observadas que con los indicadores de los otros constructos (Tabla 2).

Para los constructos emergentes (delegación, recompensas intrínsecas y recompensas extrínsecas), la Tabla 1 muestra los pesos en lugar de las cargas. El peso de un ítem representa su contribución relativa en la formación del correspondiente constructo. Es necesario tener en mente que ni se asume ni se requiere que los indicadores formativos estén correlacionados; por lo tanto, las medidas tradicionales que valoran la validez y consistencia interna no son lógicas ni apropiadas (Rodríguez Pinto, Rodríguez Escudero y Gutierrez-Cillán; 2008). Por último, se muestran las correlaciones entre las variables latentes en la Tabla 3.

El Diagrama 1 resume los resultados obtenidos al realizar el análisis PLS

Tabla 3

Correlaciones entre las variables latentes

\begin{tabular}{ccccc}
\hline & DEL & EREC & IREC & KTRS \\
\hline DEL & 1.0000 & & & \\
EREC & 0.5658 & 1.0000 & & \\
IREC & 0.4622 & 0.4544 & 1.0000 & \\
KTRS & 0.6887 & 0.6029 & 0.7169 & 1.0000 \\
\hline
\end{tabular}

Nota: $\mathrm{DEL}=$ delegación. $\mathrm{EREC}=$ recompensas extrínsecas. IREC $=$ recompensas intrínsecas. $\mathrm{KTRS}=$ transmisión de conocimiento.

Fuente: Elaboración propia.

\section{Diagrama 1}

Resultados del modelo estructural

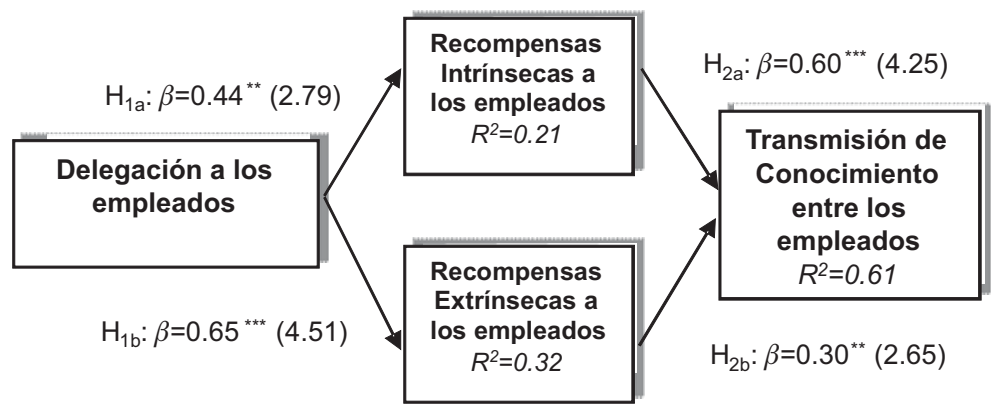

Nota: ${ }^{* * *} p<0.001 .{ }^{* *} p<0.01 .{ }^{*} p<0.05$. Valores del estadístico t se muestran entre paréntesis.

Fuente: Elaboración propia. 
para contrastar el modelo estructural, mostrando los coeficientes estandarizados $(\beta)$, el nivel de significación (estadísti$\cot$ ) y el valor del $R^{2}$ de la variable dependiente. Como se puede observar, los resultados permiten verificar la hipótesis $1 \mathrm{y}$ las dos subhipótesis derivadas de la misma: la delegación tiene un efecto significativo tanto sobre las recompensas intrínsecas $\left(\mathrm{H}_{1 \mathrm{a}}: \beta=0.44, p<0.01\right)$ como sobre las recompensas extrínsecas $\left(\mathrm{H}_{1 \mathrm{~b}}\right.$ : $\beta=0.65, p<0.001)$. Cuando aumenta la delegación -se permite que sean las personas con un mejor conocimiento las que tomen las decisiones, con independencia de la posición que ocupen dentro de la jerarquía de la organización- se hace un mayor uso de las recompensas, tanto intrínsecas como extrínsecas para alinear los objetivos individuales con los organizativos y reducir los problemas de riesgo moral, evitando el desarrollo de comportamientos oportunistas.

Teniendo en cuenta el tipo de personal que trabaja en estas organizaciones, profesionales cualificados que prestan servicios integrados a las personas con discapacidad, servicios que son complejos y requieren un conocimiento específico, resulta comprensible que se opte por delegar derechos de decisión a los individuos que tienen el conocimiento requerido para adoptar una decisión de calidad, en lugar de intentar transferir el conocimiento a aquellos que disponen de autoridad pero no de la capacidad para comprender ese conocimiento especializado. No obstante, para evitar pérdidas de control y que los empleados hagan una utilización interesada de la discrecionalidad concedida, deben ponerse en práctica sistemas de recompensas que eviten la inconsistencia de objetivos y el desarrollo de comportamientos oportunistas.

Las recompensas intrínsecas son especialmente relevantes en las organizaciones que carecen de finalidad lucrativa pero, reconociendo que no existen agentes perfectos y que los profesionales que se están considerando son muy valorados en el sector empresarial, no pueden dejarse de lado las recompensas extrínsecas, puesto que, si no se aplican en unos niveles apropiados, se corre el riesgo de experimentar unos niveles de rotación no deseables, que perjudiquen la eficiencia de la organización.

Los resultados obtenidos también confirman la hipótesis $\mathrm{H}_{2 \mathrm{a}}$ : las recompensas intrínsecas influyen positivamente sobre la transmisión de conocimiento $\left(\mathrm{H}_{2 \mathrm{a}}: \beta=0.60, p<0.001\right)$. Cuando los empleados realizan su actividad en un ambiente de respeto, su trabajo contribuye a su realización personal, se desarrolla en una organización cuyos valores comparten y que tiene unos objetivos con los cuales se identifican, aumenta su disposición a compartir su conocimiento con el resto de los empleados lo que, sin duda, contribuye a prestar una mejor atención a los beneficiarios de sus servicios. Debe tenerse presente que los servicios prestados a las personas con discapacidad -los usuarios de la organización-, suelen requerir, en la mayor parte de los casos, intervenciones complejas por las diferentes actuaciones simultáneas que tienen que realizar coordinando la actividad de diferentes profesionales con variados perfiles de formación. En este sentido, la 
Diseño organizativo de las organizaciones no lucrativas centradas en personas ... Martín-Pérez, Víctor; Martín-Cruz, Natalia y Estrada-Vaquero, Isabel

motivación intrínseca favorece la puesta en común de conocimiento tácito y reduce los problemas de oportunismo que pueden aparecer cuando se llevan a cabo actuaciones en equipo. Además, un individuo que se sienta integrado y seguro en su puesto de trabajo, tiende a disminuir el conocido como "síndrome del enemigo externo" que es una situación en la que la identificación que el individuo llega a tener con su puesto de trabajo disminuye su propensión a transmitir conocimiento para evitar que otros dispongan de sus capacidades (Argyris, 1990, 1994; Senge, 1990).

También se verifica la hipótesis $\mathrm{H}_{2 \mathrm{~b}}$ : las recompensas extrínsecas influyen positivamente sobre la transmisión de conocimiento $\left(\mathrm{H}_{2 \mathrm{~b}}\right.$ : $\left.\beta=0.30, p<0.01\right)$. Un empleado al que se ofrezca estabilidad en su trabajo, formación, flexibilidad para establecer sus horarios, permanecerá en la entidad periodos de tiempo más prolongados, incrementará su experiencia y conocimientos y su disposición para transmitirlos al resto de compañeros de trabajo. Un aspecto importante de las recompensas extrínsecas es la instrumentalidad -son un medio para lograr un fin- razón que puede llevar a los empleados a necesitar transmitir su conocimiento de un modo voluntario. Por este motivo, aunque las recompensas extrínsecas puedan no ser la principal motivación para transmitir conocimiento, son una poderosa herramienta para orientar el comportamiento de los empleados y favorecer su participación en la organización dado que cualquier trabajador se preocupa por satisfacer sus necesidades financieras para mantener un adecuado nivel de vida. Por lo tanto, si están satisfechos con las recompensas extrínsecas que proporciona su organización, serán más productivos y creativos, capaces de asumir la iniciativa y, en última instancia, estarán preparados y dispuestos para transmitir su conocimiento a otros miembros de la organización.

Los resultados obtenidos en el análisis empírico ponen de relieve que las recompensas intrínsecas se manifiestan como el elemento más importante para la transmisión de conocimiento (compartir y transmitir conocimiento dentro de la organización con otros participantes), reforzando el argumento tan extendido en la literatura de que los individuos participan en una entidad sin ánimo de lucro, fundamentalmente, por razones intrínsecas.

El modelo presentado muestra una capacidad razonable para explicar como la delegación influye sobre el uso de recompensas, tanto intrínsecas como extrínsecas, y como éstas afectan a la transmisión de conocimiento (véase el valor del $R^{2}$ para estas variables en el diagrama 1). Además, el efecto indirecto de la delegación $(\beta=0.32, p<0.001)$ sobre la transmisión de conocimiento es positivo y significativo, tal y como cabría esperar. En consecuencia, queda patente la importancia que tiene el diseño organizativo sobre la transmisión de conocimiento en las entidades sin ánimo de lucro, en este caso, las centradas en prestar servicios a personas con discapacidad.

\section{Conclusiones}

No debe perderse de vista la creciente importancia que el sector de servi- 
cios a la discapacidad ha venido adquiriendo en años recientes -sobre todo en los últimos diez-, en los que se han creado multitud de organizaciones no lucrativas en más de un centenar de países (Enns, 2008) para mejorar la calidad de vida de las personas con discapacidad, con especial énfasis en encontrar tratamientos para discapacidades poco frecuentes (laringotomización, esclerosis múltiple, síndrome de Down, autismo, lupus fatiga, entre otras), que no están suficientemente apoyadas y respaldadas por los servicios públicos, además de intentar dar voz a las personas con discapacidad -identificar sus necesidades, expresar sus prioridades, evaluar los servicios, propugnar el cambio y mejorar la concienciación pública-y ayudarles a descubrir oportunidades profesionales. Por ejemplo, en España, tanto la ley de integración social de los minusválidos (Ley 13/1982) como la ley de igualdad de oportunidades, no discriminación y accesibilidad universal de las personas con discapacidad (Ley 51/2003), han dado lugar a un entorno institucional que ha favorecido la creación de numerosas ENL de pequeña dimensión que prestan servicios en este sector, organizaciones que, en muchos casos, reciben financiación pública, lo que ha generado un creciente interés en las autoridades y en la sociedad en general por el cumplimiento de su misión.

En este contexto, cobra especial importancia un adecuado diseño organizativo que favorezca la transmisión de conocimiento por cuanto permitirá que estas pequeñas entidades sin ánimo de lucro dedicadas a la atención de personas con discapacidad puedan lograr un mejor aprovechamiento de sus muy es- casos recursos. En el presente trabajo, se sostiene que los directivos de estas pequeñas organizaciones que atienden a personas con discapacidad necesitan delegar en profesionales con un conocimiento especializado del que ellos carecen para que las decisiones técnicas adoptadas sean de mayor calidad y se contribuya a mejorar el bienestar de los usuarios a los que prestan servicios. No obstante, dado que no existen agentes perfectos, esa delegación debe venir acompañada de algún sistema de control para evitar que los empleados hagan un uso interesado de la autoridad concedida. Teniendo en cuenta que la delegación no es compatible con una excesiva supervisión directa, la percepción de recompensas vinculadas al desempeño parece el mecanismo de control más apropiado en una situación donde el conocimiento está asimétricamente distribuido.

Al aplicar sistemas de recompensas en entidades no lucrativas deben tenerse en cuenta algunas de sus características diferenciadoras. En primer lugar, aunque pueden utilizar recompensas extrínsecas, esto puede ser considerado por los donantes como una violación de la restricción de no distribución, al entender que sus recursos están siendo utilizados para pagar mayores compensaciones al personal contratado en lugar de emplearse en las necesidades que motivaron su concesión, motivo por el que se evita utilizar partidas como la asignación de bonos vinculadas al logro de resultados concretos. Por esta razón, su uso en las organizaciones del tercer sector todavía no está muy extendido y, tal y como muestran los resultados, su importancia es menor que las recompensas que no tienen un carác- 
Diseño organizativo de las organizaciones no lucrativas centradas en personas ... Martín-Pérez, Víctor; Martín-Cruz, Natalia y Estrada-Vaquero, Isabel

ter monetario. En segundo lugar, tal y como ha venido señalando la literatura, los empleados del tercer sector conceden una mayor importancia a aspectos, tales como, la implicación en la organización, identificación con su misión, sentido de pertenencia al grupo y en la toma de decisiones, así como al sector en el que opera y al modo en que la organización trabaja con sus usuarios; es decir, valoran en mayor medida las recompensas intrínsecas, lo que reduce la necesidad de recurir a recompensas extrínsecas para satisfacer su utilidad. Si bien las recompensas intrínsecas resultan más difíciles de controlar por parte de la organización -ya que, en muchos casos (autorrealización, autoconfianza, implicación, pertenencia) dependen de características personales del individuo-, debe realizarse un esfuerzo por crear el ambiente necesario para que los empleados se identifiquen con los objetivos estratégicos y con la misión de la organización, desarrollen identidades sociales y personales y, en definitiva, para que dispongan de unas condiciones de trabajo idóneas para progresar tanto en lo personal como en lo profesional.

La transmisión de conocimiento entre los empleados es un aspecto de crucial importancia para mejorar el funcionamiento de cualquier organización, más aún, si cabe, en las actividades realizadas por equipos, como es el caso de la prestación de servicios de asistencia a personas con discapacidad. Más concretamente, fisioterapeutas, psicólogos, trabajadores sociales, médicos, enfermeros y demás personal con base técnica cuentan con conocimiento especializado, por lo que necesitan trabajar conjuntamente y de forma coordinada con las personas con discapacidad a las que dirigen su atención. Este análisis se ha centrado en identificar la influencia que algunas de las más importantes variables de funcionamiento interno ejercen a la hora de facilitar la transmisión de conocimiento entre los empleados de las pequeñas ENL dedicadas a prestar servicios a estas personas.

Los resultados del estudio muestran que, tanto las recompensas extrínsecas como las intrínsecas son importantes para que los empleados incrementen su disposición a transmitir sus conocimientos al resto de compañeros de trabajo, especialmente en el caso de conocimiento tácito que es el más complejo de transmitir, y ayuden, con la experiencia adquirida, a mejorar el funcionamiento de la organización. Ahora bien, aun siendo relevantes ambos tipos de recompensas, las intrínsecas tienen una influencia significativamente mayor sobre los empleados a la hora de transferir su conocimiento y, al mismo tiempo, a captar y asumir el conocimiento de otros compañeros, contribuyendo con ello al logro de la misión de la organización.

Una recomendación importante que puede realizarse a los directivos de estas organizaciones, tomando en consideración los resultados obtenidos, es que diseñen mecanismos que permitan convertir el conocimiento tácito en explícito, de modo que la organización pueda desarrollar una memoria organizativa que facilite la eficiencia a lo largo del tiempo y reduzca el impacto negativo que pueda tener la jubilación o la salida de los empleados de la organización.

Teniendo en cuenta la carencia de recursos que tienen las ENL analizadas, 
sería altamente recomendable la creación de plataformas que permitan el intercambio interorganizativo de conocimiento y sean un punto de partida para coordinar sus esfuerzos en la búsqueda de financiación o en otras actividades de apoyo.

\section{Referencias bibliográficas}

Anderson, James C, y Gerbing, David W. (1988). An update paradigm for scale development incorporating unidimensionality and its assessment. Journal of Marketing Research, Volumen 25, No 2, USA, American Marketing Association, pp 186-192.

Argirys, Chris (1990). Overcoming organizational defenses. USA, Needham Heights, Massachussets, Allyn \& Bacon.

Argirys, Chris (1994). Good communications that blocks learning. Harvard Business Review, Volumen 72, No 3 , USA, Harvard Business School, pp 7-85.

Balkin, David B. y Gomez-Mejia, Luis R. (1990). Matching compensation and organizational strategies. Strategic Management Journal, Volumen 11, No 2, USA, Strategic Management Society, pp 153-169.

Barnabé, Clermont y Burns, Mirlded (1994). Teachers job characteristics and motivation. Educational Research, Volumen 36, No 2, United Kingdom, National Foundation for Educational Research, pp 171-185.

Bartol, Kathryn M. y Srivastava, Abhishek (2002). Encouraging knowledge sharing: The role of organizational reward systems. Journal of Leadership and Organization Studies, Volumen 9, No 1, USA, Midwest Academy of Management, pp 64-76.
Bock, Gee-Woo; Zmud, Robert W; Kim, Young-Gul y Lee, Jae-Nam (2005). Behavioral intention formation in knowledge sharing: Examining the roles of extrinsic motivator, socialpsychological forces and organizational climate. MIS Quarterly, Volumen 9, No 1, USA, University of Minnesota, MIS Research Center, pp 87-111.

Bonner, Sarah E. y Sprinkle, Geoffrey B. (2002). The effects of monetary incentives on effort and task performance: Theories, evidence, and a framework for research. Accounting, Organizations and Society, Volumen 27, No 4-5, United Kingdom, Saïd Business School, Oxford University, pp 303-45.

Brickley, James A; Smith, Clifford W. y Zimmerman, Jerold L. (1995). The economics of organizational architecture. Journal of Applied Corporate Finance, Volumen 8, No 2, Morgan Stanley, pp 19-31.

Brickley, James A. y Van Horn, Lawrence R. (2002). Managerial incentives in nonprofit organizations: Evidence from hospitals. Journal of Law and Economics, Volumen 45, No 1, USA, University of Chicago Law School, pp 227-249.

Burgess, Diana (2005). What motivates employees to transfer knowledge outside their work unit? Journal of Business Communication, Volumen 42, No 4, USA, Association of Business Communication, pp 324-48.

Callen, Jeffrey L. y Falk, Haim (1993). Agency and efficiency in nonprofit organizations: The case of "specific health focus" charities. The Accounting Review, Volumen 68, No 1, USA, American Accounting Association, pp 4865.

Christie, Andrew A; Joye Marc P. y Watts, Ross L. (2003). Decentralization of the firm. Theory and evidence. Journal of 
Diseño organizativo de las organizaciones no lucrativas centradas en personas ...

Martín-Pérez, Víctor; Martín-Cruz, Natalia y Estrada-Vaquero, Isabel

Corporate Finance, Volumen 9, No 2, England, Elsevier Science Ltd., pp 3-36.

Colella, Adrienne (2001). Coworker distributive fairness judgments of the workplace accommodation of employees with disabilities. Academy of Management Review, Volumen 26, No 1, USA, Academy of Management, pp 100-116.

Collins, Robert A. y Yeager, John L. (1988). Staff evaluation and incentive practices utilized by behavioral science research organizations: A pilot study. Journal of the Society of Research Administrators, Volumen 20, No 1, USA, Society of Research Organizations International, pp 119-129.

De Gieter, Sara; De Cooman, Rein; Pepermans, Roland; Caers, Ralf; Du Bois, Cindy y Jegers, Marc (2006). Identifying nurses' rewards: A qualitative categorization study in Belgium. Human Resources for Health, Volumen 15, No 4, World Health Organization, pp 1-8.

Delaney, John T. y Mark A. Huselid (1996). The impact of human resource management practices on perceptions of organizational performance. Academy of Management Journal, Volumen 39, No 4, USA, Academy of Management, pp 949-969.

Enns, Henry (2008). The role of organizations of disabled people. A Disabled Peoples' International Discussion Paper, Sweden, Independent Living Institute.

Fornel, Claes y Larcker, David F. (1981). Structural equation models with unobservable variables and measurement error: Algebra and statitics. Journal of Marketing Research, 25(2), USA, American Marketing Association, pp 186-192.
Gordon, Lawrence A. y Narayanan, Vadake K. (1984). Management accounting systems, perceived environmental uncertainty and organization structure: An empirical investigation. Accounting, Organizations and Society, Volumen 9, No 1, United Kingdom, Saïd Business School, Oxford University, pp 33-47.

Hallock, Kevin F. (2002). Managerial pay and governance in American nonprofits. Industrial Relations, Volumen 41, No 3, USA, Institute for Research on Labor and Employment, University of California, Berkeley, pp 377-406.

Handy, Femida y Katz, Eliakim (1998). The wage differential between nonprofit institutions and Corporations: Getting more by paying less? Journal of Comparative Economics, Volumen 26, No 2, USA, Association for Comparative Economic Studies, pp 246-261.

Hansmann, Henry B. (1980). The role of nonprofit enterprise, Yale Law Journal, Volumen 89, No 5, USA, Yale Law Journal Company, Inc., pp 835-901.

Hayek, Friedrich A. (1945). The use of scientific knowledge in society. American Economic Review, Volumen 35, No 4, USA, American Economic Association, pp 519-530.

Hendriks, Paul (1999). Why share knowledge? The influence of ICT on the motivation for knowledge sharing. Knowledge and Process Management, Volumen 6, No 2, USA, Wiley Periodicals Inc., pp 91-100.

Huysman, Marleen y de Wit, Dick (2004). Practices of managing knowledge sharing: Towards a second wave of knowledge management. Knowledge and Process Management, Volumen 11, No 2, USA, Wiley Periodicals Inc., pp 81-92.

Jensen, Michael C. y Meckling, William $H$. (1976). Theory of the firm: Managerial 
behavior, agency costs, and ownership structure. Journal of Financial Economics, Volumen 3, No 4, The Netherlands, Graduate School of Business Administration, Elsevier Science B.V., pp 305-360.

Jensen, Michael C. y Meckling, William H. (1992). Specific and general knowledge and organizational structure. In Lars Werin and Hans Wijkander (Eds.) Contract economics, Oxford: Blackwell, pp 251-274.

Ko, Dong-Gil; Kirsch, Laurie J. y King, William R. (2005). Antecedents of knowledge transfer from consultants to clients in enterprise system implementations, MIS Quarterly, Volumen 29, No 1, USA, University of Minnesota, MIS Research Center, pp 59-85.

Kofman, Fred y Senge, Peter M. (1993). Communities of commitment: The heart of learning organizations. Organizational Dynamics, USA, Elsevier Science Ltd., Volumen 22, No 2, pp 5-23.

Kogut, Bruce y Zander, Udo (1996). What firms do? Coordination, identity, and learning, Organization Science, Volumen 7, No 5, USA, Institute for Operations Research and the Management Sciences, pp 502-518.

Leete, Laura (2000). Wage equity y employee motivation in nonprofit and for-profit organizations. Journal of Economic Behavior and Organization, Volumen 43, No 4, The Netherlands, Elsevier Sequoia S.A., pp 423-446.

Lucas, Leyland M. y Ogilvie, D. (2006). Things are not always what they seem. How reputations, culture and incentives influence knowledge transfer. The Learning Organization, Volumen 13, No 1, England, Emerald Group Publishing Limited, pp 7-24.

Martín-Cruz, Natalia; Martín-Pérez, Víctor y Trevilla-Cantero, Celina (2009). The influence of employee motivation on knowledge transfer. Journal of Knowledge Management, Volumen 13, No 6, England, Emerald Group Publishing Limited, pp 478-490.

Martín-Pérez, Víctor; Martín-Cruz, Natalia y Hernangómez-Barahona, Juan (2007). La construcción de la arquitectura organizativa en las entidades sin fines de lucro. El caso de las ONGD en España. Investigaciones Europeas de Dirección y Economía de la Empresa, Volumen 14, No 1, España, Academia Europea de Dirección y Economía de la Empresa, pp 51-72.

Martín-Pérez, Víctor; Martín-Cruz, Natalia y Hernangómez-Barahona, Juan (2010). Delegación y sistemas de incentivos en las entidades sin fines de lucro. El caso de las ONGD en España. Revista Europea de Dirección y Economía de la Empresa, Volumen 19, No 4, España, Academia Europea de Dirección y Economía de la Empresa, pp 171-190.

Milgrom, Paul y Roberts, John (1992). Economics, organization, and management. USA, Englewood Cliffs, CO: Prentice-Hall.

Mirvis, Philip H. y Hackett, Edward J. (1983). Work and work force characteristics in the nonprofit sector. Monthly Labor Review, Volumen 106, No 4, USA, Bureau of Labor Statistics, pp 3-12.

O'Connor, Neale G; Deng, Johny y Luo, Yadong (2006). Political constraints, organization design and performance measurement in China's state-owned enterprises. Accounting, Organizations and Society, Volumen 31, No 2, United Kingdom, Saïd Business School, Oxford University, pp 157-177.

Osterloh, Margit y Frey, Bruno (2000). Motivation, knowledge transfer and organizational forms. Organization Science, Volumen 1, No 5, USA, Institute 
Diseño organizativo de las organizaciones no lucrativas centradas en personas ...

Martín-Pérez, Víctor; Martín-Cruz, Natalia y Estrada-Vaquero, Isabel

for Operations Research and the Management Sciences, pp 538-550.

Pedraja-Rejas, Liliana; Rodríguez-Ponce, Emilio y Rodríguez-Ponce, Juan (2009). Gestión del conocimiento, eficacia organizacional en pequeñas y medianas empresas. Revista Venezolana de Gerencia, Volumen 14, No 48, Venezuela, Centro de Estudios de la Empresa, Universidad del Zulia, pp 495-506.

Pfeffer, Jeffrey (1998). Six dangerous myths about pay. Harvard Business Review, Volumen 76, No 3, USA, Harvard Business School, pp 109-119.

Prendergast, Canice (1999). The provision of incentives in firms. Journal of Economic Literature, Volumen 7, No 1, USA, American Economic Association, pp 7-63.

Preston, Anne (1989). The nonprofit worker in a for-profit world. Journal of Labor Economics, Volumen 7, No 4, USA, University of Chicago, pp 438-463.

Preyra, Colin y Pink, George (2001). Balancing incentives in the compensation contracts of nonprofits hospital CEOs. Journal of Health Economics, Volumen 20, No 4, International Health Economics Association, pp 509-525.

Quigley, Narda R; Tesluk, Paul E; Locke, Edwin A. y Bartol, Kathryn M. (2007). Multilevel investigation of the motivational mechanisms underlying knowledge sharing and performance. Organization Science, Volumen 18, No 1, USA, Institute for Operations Research and the Management Sciences, pp 71-92.

Ringle, Christian M; Wende, Sven y Will, Alexander (2005). Customer segmentation with FIMIX-PLS. In T. Aluja, V. Esposito Vinzi, J. Casanovas, A. Morineau, and $\mathrm{M}$. Tenenhaus (Eds.), PLS and related methods, Proceedings of the PLS'05 International Sympo- sium, SPAD Testandgo, Paris, pp 507-514.

Rodríguez-Pinto, Javier; Rodríguez-Escudero, Ana I. y Gutiérrez-Cillán, Jesús. (2008). Order, positioning, scope and outcomes of market entry. Industrial Marketing Management, Volumen 37, No 2, USA, American Marketing Association, pp 154-166.

Roomkin, Myron J. y Weisbrod, Burton A. (1999). Managerial compensation and incentives in for-profit and nonprofit hospitals, Journal of Law, Economics and Organization, Volumen 15, No 3, United Kingdom, Oxford University Press, pp 750-781.

Rose-Ackerman, Susan (1986). The economics of nonprofit institutions. USA, NY: Oxford University Press.

Ruhm, Christopher J. y Borkoski, Carey (2003). Compensation in the nonprofit sector. Journal of Human Resources, Volumen 38, No 4, USA, University of Winconsin Press, pp 992-1021.

Senge, Peter (1990). The fifth discipline, USA, New York, Doubleday Publisher.

Slater, Stanley F. y Narver, John C. (1995). Market orientation and the learning organization. Journal of Marketing, Volumen 59, No 3, USA, American Marketing Association, pp 63-74.

Steinberg, Richard (1990). Labor economics and the nonprofit sector: A literature review. Nonprofit and Voluntary Sector Quarterly, Volumen 19, No 2, USA, Association for Research on Nonprofit Organizations and Voluntary Action, pp 151-169.

Stone, Dianna L. y Colella, Adrienne (1996). A model of factors affecting the treatment of disabled individuals in Organizations. Academy of Management Review, Volumen 21, No 2, USA, 
Academy of Management, pp 352401.

Swieringa, Joop y Wierdsma, Andre (1992). Becoming a learning organization: Beyond the Learning Curve. USA, Addison-Wesley, Reading: MA.

Tampoe, Mahen (1993). Motivating knowledge employees: The challenge for the 1990s. Longe Range Planning, Volumen 26, No 3, United Kingdom, Strategic Planning Society and European Strategic Planning Federation, pp 49-55.

Theuvsen, Ludwig (2004). Doing better while doing good: Motivational aspects of pay-for-performance effectiveness in nonprofit organizations. International Journal of Voluntary and Nonprofit Organizations, Volumen 5, No 2, USA, the International Society for Third-Sector Research, pp 17-136.

Tortia, Ermanno C. (2008). Worker well-being and perceived fairness: Survey-based findings from Italy. The Journal of Socio-Economics, Volumen 37, No 5, England, Elsevier Science Ltd., pp 2080-2094.
Tyler, Tom (2003). Trust within organizations, Personnel Review, Volumen 32, No 5, England, Emerald Group Publishing, pp 556-68.

Young, Dennis R. y Finch, Stephen J. (1977). Foster care and nonprofit agencies. USA, Lexington Books, MA: D.C. Heath and Company.

Young, Dennis R. (1983). If not for profit, for what?. USA, Lexington, MA: Lexington Books.

Widener, Sally K; Shackell, Margaret B. y Demers, Elizabeth A. (2008). The juxtaposition of social surveillance controls with traditional organizational design components. Contemporary Accounting Research, Volumen 25, No 2, Canada, The Canadian Academic Accounting Association, pp 605-638.

Zárraga, Celia y Bonache, Jaime (2003). The impact of team atmosphere on knowledge outcomes in self-managed teams. Organization Science, Volumen 26, No 5, USA, Institute for Operations Research and the Management Sciences, pp 1227-1245. 